\title{
Unveiling the Role of the Integrated Endoplasmic Reticulum Stress Response in Leishmania Infection - Future Perspectives
}

\author{
K. L. Dias-Teixeira', R. M. Pereira ${ }^{2}$, J. S. Silva ${ }^{3}$, N. Fasel', B. H. Aktas ${ }^{5}$ and U. G. Lopes ${ }^{1 *}$ \\ ${ }^{1}$ Institute of Biophysics Carlos Chagas Filho, Federal University of Rio de Janeiro, Rio de Janeiro, Brazil, ${ }^{2}$ Institute of \\ Microbiology Paulo de Goes, Federal University of Rio de Janeiro, Rio de Janeiro, Brazil, ${ }^{3}$ Department of Biochemistry and \\ Immunology, University of São Paulo, Ribeirão Preto, Brazil, ${ }^{4}$ Department of Biochemistry, Faculty of Biology and Medicine, \\ Center for Immunity and Infection Lausanne, University of Lausanne, Lausanne, Switzerland, ${ }^{5}$ Laboratory of Translation, \\ Department of Hematology, Brigham and Women's Hospital, Harvard Medical School, Boston, MA, USA
}

\section{OPEN ACCESS}

Edited by: Abhay Satoskar, Ohio State University, USA

Reviewed by: Patricia Talamás-Rohana, Cinvestav, Mexico Ranadhir Dey, Food and Drug Administration, USA

${ }^{*}$ Correspondence: U. G. Lopes lopesu@biof.ufri.br

Specialty section: This article was submitted to Microbial Immunology, a section of the journal Frontiers in Immunology

Received: 13 May 2016 Accepted: 13 July 2016 Published: 22 July 2016

Citation:

Dias-Teixeira KL, Pereira RM,

Silva JS, Fasel N, Aktas BH and Lopes UG (2016) Unveiling the Role of the Integrated Endoplasmic Reticulum Stress Response in Leishmania Infection - Future Perspectives.

Front. Immunol. 7:283. doi: 10.3389/fimmu.2016.00283
The integrated endoplasmic reticulum stress response (IERSR) is an evolutionarily conserved adaptive mechanism that ensures endoplasmic reticulum (ER) homeostasis and cellular survival in the presence of stress including nutrient deprivation, hypoxia, and imbalance of $\mathrm{Ca}^{+}$homeostasis, toxins, and microbial infection. Three transmembrane proteins regulate integrated signaling pathways that comprise the IERSR, namely, IRE-1 that activates XBP-1, the pancreatic ER kinase (PERK) that phosphorylates the eukaryotic translation initiation factor 2 and transcription factor 6 (ATF6). The roles of IRE-1, PERK, and ATF4 in viral and some bacterial infections are well characterized. The role of IERSR in infections by intracellular parasites is still poorly understood, although one could anticipate that IERSR may play an important role on the host's cell response. Recently, our group reported the important aspects of XBP-1 activation in Leishmania amazonensis infection. It is, however, necessary to address the relevance of the other IERSR branches, together with the possible role of IERSR in infections by other Leishmania species, and furthermore, to pursue the possible implications in the pathogenesis and control of parasite replication in macrophages.

Keywords: Leishmania, ER stress, XBP-1, IFN-1, PERK, ATF4

\section{INTRODUCTION}

The endoplasmic reticulum (ER) is a dynamic tubular network involved in different processes such as protein folding, lipid synthesis, and the biogenesis of autophagosomes and peroxisomes (1). When the process of protein synthesis and/or folding is disturbed, the ER induces a transcriptional program, the integrated endoplasmic reticulum stress response (IERSR), leading to the increase of ER-chaperone expression, lipid synthesis, and the induction of other sets of gene products involved in the retrograde transport and degradation of unfolded proteins (ERAD) (2). These conserved adaptive responses reduce demand on the folding capacity or ER, increase ER's folding capacity, and clear this organelle off of unfolded proteins. However, during this process, a set of genes that also regulate the expression of cytokines and promote the resistance to oxidative stress are upregulated. The three branches that regulate the ER response are comprised by the activating transcription factor 6 (ATF6), inositol-requiring kinase 1 (IRE-1), and the protein kinase R (PKR)-like endoplasmic reticulum kinase (PERK). IRE-1 activates the X-box binding protein 1 (XBP-1), a transcriptional 
factor that plays a critical role in cellular homeostasis and regulates the expression of important cytokines related to the antiviral immunity response, such as IFN1- $\beta$. PERK phosphorylates eIF2 $\alpha$, which reduces overall protein synthesis while upregulating the expression of activating transcription factor 4 (ATF4), which drives the expression of genes that play a critical role in restoring cellular homeostasis, resistance to oxidative stress together with genes related to the autophagic pathway and the innate immunity response. Interestingly, both XBP-1 and ATF4 can be activated by toll-like receptors (TLRs). For instance, the engagement of TLR2 and TLR4 can specifically activate XBP-1 leading to the production of pro-inflammatory cytokines that restrain bacterial burden in infected macrophages (3). ATF4 can be directly activated by the TLR4-MyD88 pathway following stimulation of human monocytes with lipopolysaccharide (LPS) (4).

Viruses can selectively induce specific branches of the IERSR. For instances, human cytomegalovirus and hepatitis $\mathrm{C}$ activate the IERSR response, while some viruses, such as dengue virus and hepatitis $\mathrm{C}$ virus induce the IERSR trough the exploitation of the ER membranes during the replication process (5). Additionally, some viruses induce the IERSR and the inhibition of the translational process due to the phosphorylation of eIF2- $\alpha$, reducing the production of cytokines and interfering with the host immune response. This process is highly induced by enteroviruses (6). Some viruses adapted the IERSR pathways to favor their infection directly. The phosphorylation of eIF2- $\alpha$ induces the translation of a specific set of proteins including ATF4. ATF4 can, for example, enhance human immunodeficiency virus (HIV) replication through a synergistic interaction with the HIV regulatory protein Tat (7).

The role of IERSR pathways in parasite infection is poorly investigated. Recently, it was reported that Plasmodium berghei induces the ER stress response and XBP-1 mRNA splicing and translation of the transcriptionally active XBP-1 spliced form (XBP-1s) in hepatocytes. This activation was demonstrated to be crucial for parasite replication inside hepatocytes and to the progression of the infection (8). XBP-1s can modulate the synthesis of phospholipids, such as phosphatidylcholine (PC), in hepatocytes. PC is a major component of membranes, and it has been demonstrated that malaria parasites uptake host-derived PC and, most probably, PC is also employed for enlarging the parasitophorous vacuole membrane (9). Most recently, we showed that induction of ER stress favors Leishmania amazonensis infection in a TLR2-dependent manner, culminating in the formation of $\mathrm{XBP}-1$ s. XBP- 1 induces IFN- $\beta$ expression and modulates the oxidative response of infected macrophages, thereby promoting parasite proliferation (10).

However, it will be important to test these observations in other Leishmania species and to address the relevance of the PERK/ATF4 and ATF6 branches of the IERSR during Leishmania infection.

\section{THE ROLE OF XBP-1 IN LEISHMANIA INFECTION}

We recently observed that $L$. amazonensis induces the activation of XBP-1 in macrophages. RAW 264.7 cells knocked down for XBP-1 exhibited reduced parasite load, likely due to impaired translocation of the IRF3 transcription factor resulting in reduced IFN-1 expression (10). We also observed that infected XBP-1 knocked down macrophages produce higher nitric oxide levels and reduced Hemeoxygenase ( $\mathrm{HO})-1$ expression compared to control macrophages. However, how XBP-1 controls oxidative stress in L. amazonensis infection requires further investigation. One mechanism that could induce this effect is the activation or repression of the NF- $\mathrm{KB}$ transcription factor. $L$ amazonensis activates an NF- $\mathrm{KB}$ p50/p50 repressor homodimer, which promotes reduction in iNOS expression and favors parasite growth (11). The production of ROS can activate the ER stress response, which can suppress $\mathrm{NF}-\kappa \mathrm{B}$ activation in the later phase of IERSR (12). The protein A20, an ubiquitin-editing NF- $\kappa B$ inhibitor protein, may play an important role in this process, as this protein can negatively regulate $\mathrm{NF}-\kappa \mathrm{B}$ during oxidative stress (13). Additionally, it is important to understand if other Leishmania species induce the IERSR branches, and the role, if any, in pathogenesis. Experiments carried out by our group observed an induction of the XBP-1 spliced form in clinical samples from patients infected with Leishmania braziliensis, another Leishmania species widely found in Brazil and the main causative agent of cutaneous leishmaniasis. These data indicate that other Leishmania species can activate this pathway, and that IERSR may play a role in Leishmania-associated pathogenesis.

\section{THE INDUCTION OF ER STRESS: THE ROLE OF TLRs in XBP-1 ACTIVATION IN LEISHMANIA INFECTION}

The mechanism through which L. amazonensis induces ER stress is not understood. Leishmania parasitophorous vacuoles interact continuously with the ER compartment and may recruit components that are important for parasite intracellular survival (14). The inhibition of such membrane compartment fusion with the parasitophorous vacuole results in the reduction of infection (15). It is conceivable that such compartment fusions may favor the activation of IERSR branches in infection.

The contribution of TLR receptors in IERSR remains to be elucidated. There is evidence to suggest that TLRs play a role for the success of L. amazonensis infection that is linked with IERSR activation. For instance, when TLR2 KO macrophages were treated with the ER stress inductor thapsigargin, there was a reduction of the L. amazonensis proliferation compared to wildtype cells (10). Additional results obtained by our group showed that TLR2 was partially required for XBP-1 activation (splicing) due to L. amazonensis infection. However, the mechanism by which $L$. amazonensis induces XBP-1 activation and ER stress remains unclear.

\section{THE PERK/ATF4 BRANCH OF IERSR and LEISHMANIA INFECTION: IS A FUNCTIONAL ROLE?}

The PERK/ATF4 branch of IERSR plays an important role in certain cellular processes that are also exploited to establish Leishmania infection. For instance, L. amazonensis induces the 
PI3K/AKT signaling pathway (16), and it has been reported that the PERK-eIF2 $\alpha$ pathway and PI3K signaling increases ATF4 expression, nuclear localization, and transcriptional activity (17-19). Additionally, PERK can directly regulate the activation of the nuclear factor (erythroid-derived 2)-like 2 (NRF2), an important antioxidant transcription factor that regulates the expression of a number of antioxidative response genes (20). Additionally, ATF4 has an important role in the autophagy. PERK/ eIF2 $\alpha /$ ATF4 signaling can induce upregulation of cytoprotective autophagy genes, such as ATG5 and ATG7, which promote cellular survival (21). In addition, ATF4 controls the microtubule associated protein 1A/1B-light chain 3 (LC3) expression. LC3B is important to generate the autophagosome formation, a hallmark of the autophagic process $(22,23)$. In 2012, Cyrino et al. showed that Leishmania parasites induce LC3B conversion and suggested that autophagy favors L. amazonensis infection (24). ATF4 is upregulated by HIV-1 infection and enhances HIV replication, likely due to synergistic interactions with the HIV Tat protein. Importantly, the expression of ATF4 induces HIV reactivation in chronically infected cell lines (7). Recently, our group showed that the Tat viral protein also increases L. amazonensis infection, in a PKR-dependent manner (25). L. amazonensis is able to induce PKR, a pathway activated in viral infections (26). L. amazonensis can also modulate IFN-1 expression in a TLR2/ PKR-dependent fashion to promote the infection by the parasite, another pathway that is shared in viral infections (27). Taken together, due to classical function of IERSR in viral infections, it is relevant to test the role of PERK/ATF4 in viral co-infection and Leishmania.

\section{CONCLUSION REMARKS}

It is well known that the IERSR can modulate viral and bacterial infection, promoting the induction of cytokines, including IFN-1, which can be determinant to the outcome of several infections. Recent work suggests that the IERSR is required for the development of intracellular parasites. For instance, the activation of XBP-1 in hepatocytes infected by $P$. berghei favor the infection by the parasite through the modulation of lipid synthesis. Corroborating this notion, it has been demonstrated that L. amazonensis activates XBP-1 leading to IFN-1 expression and the expression of antioxidative responsive genes, such as HO-1. Unveiling the mechanisms by which IERSR promote intracellular parasitic infection requires further investigation. These investigations would include determining the role of XBP-1 in resistance to oxidative stress due to Leishmania infection and examining other components of the ER stress signaling pathway,

\section{REFERENCES}

1. Hetz C. The unfolded protein response: controlling cell fate decisions under ER stress and beyond. Nat Rev Mol Cell Biol (2012) 3(2):89-102. doi:10.1038/nrm3270

2. Martinon F, Glimcher LH. Regulation of innate immunity by signaling pathways emerging from the endoplasmic reticulum. Curr Opin Immunol (2011) 23(1):35-40. doi:10.1016/j.coi.2010.10.016

3. Martinon F, Chen X, Lee AH, Glimcher LH. TLR activation of the transcription factor XBP1 regulates innate immune responses in macrophages. Nat Immunol (2010) 11(5):411-8. doi:10.1038/ni.1857

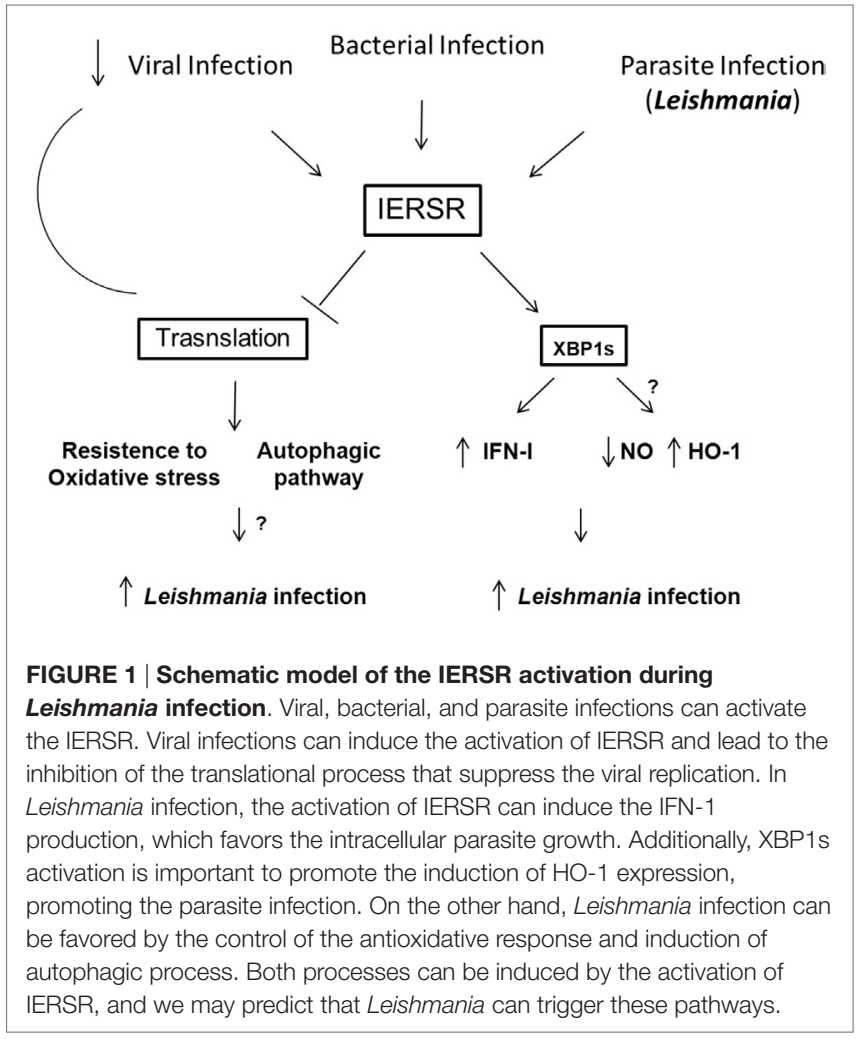

such as ATF6, in the context of parasitic infection. We can predict that the investigation of IERSR in intracellular parasitic infections may reveal novel drug targets. Figure 1 shows the a schematic model of IERSR activation in Leishmania infection.

\section{AUTHOR CONTRIBUTIONS}

$\mathrm{UL}$ - project supervisor and wrote the paper, KD-T - performed experiments and wrote the paper, RP - revised the paper and discussed the results, JS - provided samples and supervised experiments, NF - revised the paper and contributed with the discussion, BA - supervised experiments, revised the paper, and discussed results.

\section{FUNDING}

This work was supported by the Conselho Nacional de Desenvolvimento Científico e Tecnológico, Brazil, to UL. 
7. Caselli E, Benedetti S, Gentili V, Grigolato J, Di Luca D. Short communication: activating transcription factor 4 (ATF4) promotes HIV type 1 activation. AIDS Res Hum Retroviruses (2012) 28(8):907-12. doi:10.1089/AID.2011.0252

8. Inácio P, Zuzarte-Luís V, Ruivo MT, Falkard B, Nagaraj N, Rooijers K, et al. Parasite-induced ER stress response in hepatocytes facilitates Plasmodium liver stage infection. EMBO Rep (2015) 16(8):955-64. doi:10.15252/ embr.201439979

9. Itoe MA, Sampaio JL, Cabal GG, Real E, Zuzarte-Luis V, March S, et al. Host cell phosphatidylcholine is a key mediator of malaria parasite survival during liver stage infection. Cell Host Microbe (2014) 16(6):778-86. doi:10.1016/j. chom.2014.11.006

10. Dias-Teixeira KL, Calegari-Silva TC, Dos Santos GR, Vitorino Dos Santos J, Lima C, Medina JM, et al. The integrated endoplasmic reticulum stress response in Leishmania amazonensis macrophage infection: the role of X-box binding protein 1 transcription factor. FASEB J (2016) 30(4):1557-65. doi:10.1096/f.15-281550

11. Calegari-Silva TC, Pereira RM, De-Melo LD, Saraiva EM, Soares DC, Bellio $\mathrm{M}$, et al. NF-kappaB-mediated repression of iNOS expression in Leishmania amazonensis macrophage infection. Immunol Lett (2009) 127(1):19-26. doi:10.1016/j.imlet.2009.08.009

12. Kitamura M, Hiramatsu N. The oxidative stress: endoplasmic reticulum stress axis in cadmium toxicity. Biometals (2010) 23(5):941-50. doi:10.1007/ s10534-010-9296-2

13. Nakajima S, Kitamura M. Bidirectional regulation of NF- $\kappa$ B by reactive oxygen species: a role of unfolded protein response. Free Radic Biol Med (2013) 65:162-74. doi:10.1016/j.freeradbiomed.2013.06.020

14. Ndjamen B, Kang BH, Hatsuzawa K, Kima PE. Leishmania parasitophorous vacuoles interact continuously with the host cell's endoplasmic reticulum; parasitophorous vacuoles are hybrid compartments. Cell Microbiol (2010) 12(10):1480-94. doi:10.1111/j.1462-5822.2010.01483.x

15. Canton J, Ndjamen B, Hatsuzawa K, Kima PE. Disruption of the fusion of Leishmania parasitophorous vacuoles with ER vesicles results in the control of the infection. Cell Microbiol (2012) 14(6):937-48. doi:10.1111/j.14625822.2012.01767.x

16. Calegari-Silva TC, Vivarini ÁC, Miqueline M, Dos Santos GR, Teixeira KL, Saliba AM, et al. The human parasite Leishmania amazonensis downregulates iNOS expression via NF- $\mathrm{KB}$ p50/p50 homodimer: role of the PI3K/Akt pathway. Open Biol (2015) 5(9):150118. doi:10.1098/rsob.150118

17. Cao H, Yu S, Yao Z, Galson DL, Jiang Y, Zhang X, et al. Activating transcription factor 4 regulates osteoclast differentiation in mice. J Clin Invest (2010) 120:2755-66. doi:10.1172/JCI42106

18. Inageda K. Insulin modulates induction of glucose-regulated protein 78 during endoplasmic reticulum stress via augmentation of ATF4 expression in human neuroblastoma cells. FEBS Lett (2010) 584:3649-54. doi:10.1016/j. febslet.2010.07.040
19. Lian N, Lin T, Liu W, Wang W, Li L, Sun S, et al. Transforming growth factor $\beta$ suppresses osteoblast differentiation via the vimentin activating transcription factor 4 (ATF4) axis. JBiol Chem (2012) 287:35975-84. doi:10.1074/jbc. M112.372458

20. Dey S, Sayers CM, Verginadis II, Lehman SL, Cheng Y, Cerniglia GJ, et al. ATF4-dependent induction of hemeoxygenase 1 prevents anoikis and promotes metastasis. J Clin Invest (2015) 125(7):2592-608. doi:10.1172/ JCI78031

21. Luo J-Q, Chen D-W, Yu B. Upregulation of amino acid transporter expression induced by L-leucine availability in L6 myotubes is associated with ATF4 signaling through mTORC1-dependent mechanism. Nutrition (2013) 29:284-90. doi:10.1016/j.nut.2012.05.008

22. Kabeya Y, Mizushima N, Ueno T, Yamamoto A, Kirisako T, Noda T, et al. LC3, a mammalian homologue of yeast Apg8p, is localized in autophagosome membranes after processing. EMBO J (2000) 19(21):5720-8. doi:10.1093/ emboj/19.21.5720

23. Baehrecke EH. Autophagy: dual roles in life and death? Nat Rev Mol Cell Biol (2005) 6(6):505-10. doi:10.1038/nrm1666

24. Cyrino LT, Araújo AP, Joazeiro PP, Vicente CP, Giorgio S. In vivo and in vitro Leishmania amazonensis infection induces autophagy in macrophages. Tissue Cell (2012) 44(6):401-8. doi:10.1016/j.tice.2012.08.003

25. Vivarini Áde C, Pereira Rde M, Barreto-de-Souza V, Temerozo JR, Soares DC, Saraiva EM, et al. HIV-1 Tat protein enhances the intracellular growth of Leishmania amazonensis via the ds-RNA induced protein PKR. Sci Rep (2015) 5:16777. doi:10.1038/srep16777

26. Pereira RM, Teixeira KL, Barreto-de-Souza V, Calegari-Silva TC, De-Melo LD, Soares DC, et al. Novel role for the double-stranded RNA-activated protein kinase PKR: modulation of macrophage infection by the protozoan parasite Leishmania. FASEB J (2010) 24(2):617-26. doi:10.1096/ff.09-140053

27. Vivarini Ade C, Pereira RM, Teixeira KL, Calegari-Silva TC, Bellio M, Laurenti $\mathrm{MD}$, et al. Human cutaneous leishmaniasis: interferon-dependent expression of double-stranded RNA - dependent protein kinase (PKR) via TLR2. FASEB $J(2011)$ 25(12):4162-73. doi:10.1096/fj.11-185165

Conflict of Interest Statement: The authors declare that the research was conducted in the absence of any commercial or financial relationships that could be construed as a potential conflict of interest.

Copyright (c) 2016 Dias-Teixeira, Pereira, Silva, Fasel, Aktas and Lopes. This is an open-access article distributed under the terms of the Creative Commons Attribution License (CC BY). The use, distribution or reproduction in other forums is permitted, provided the original author(s) or licensor are credited and that the original publication in this journal is cited, in accordance with accepted academic practice. No use, distribution or reproduction is permitted which does not comply with these terms. 